\title{
Kapitel 1. \\ Einführung
}

\begin{abstract}
Von den alten ist Nonnus, sonsten ein gutter Christ, noch fürhanden; der in Griechischer sprachen, welche dann trefflich wol hierzu dienet, acht und viertzig Bücher, Dionysiaca genennet, geschrieben hat.
\end{abstract}

Daniel Heinsius ${ }^{1}$

Un manteau Tyrian fécouloit sur tes hanches, Un chappelet de liz mellés de roses franches, Et de feueille de vigne, \& de lhierre espars, Voltigeant, umbrageoit ton chef de tout pars.

Pierre de Ronsard ${ }^{2}$

\subsection{Nonnos und die Dionysiaka}

Die Dionysiaka sind das letzte große Literaturwerk der Antike, zu gleichen Teilen Renaissance, Zenit und Finale des griechischen Epos. Das 48 Bücher umfassende opus magnum, „die letzte große Dichtung, die wir aus der Antike erhalten haben“"3, schildert die Geschichte des Zeus-Sohnes Dionysos von seiner außergewöhnlichen Geburt aus dem Schenkel seines Vaters über seine Kindheitserlebnisse, seine Bewährungsproben in Kriegs- und Friedenszeiten bis zu seiner letztendlichen Aufnahme in den olympischen Götterhimmel. Es bietet eine größtmögliche räumliche Ausdehnung, deckt mit seinen Orten und Landschaften den gesamten griechischsprachigen Kulturkreis ab und gibt ferner den Ausblick auf phantastische, fernöstliche Gebiete frei, die seit dem Alexander-Zug und der damit verbundenen Literatur in den Wahrnehmungshorizont der Hellenen fielen. ${ }^{4}$ Die Dionysiaka bieten außerdem eine schier unüberblickbare Zahl an Mythen auf, die den Gott und das dionysische Prinzip sowohl in seiner Totalität als auch in all seinen einzelnen Ausprägungen erfassen. Nicht selten handelt es sich um Mythen, die lediglich lose mit Dionysos in Verbindung stehen oder aber sich zunächst so ganz und gar nicht in die Erzähllinie um den Gott und seinen Kult fügen wollen.

Nonnos von Panopolis liefert in vielfacher Hinsicht ein Universalepos, in dem er sämtliche mythischen und literarischen Traditionen kumuliert und Resümee über die

1 Opitz (1622), aus der Vorrede.

2 de Ronsard u. Dorat (1555) $12 \mathrm{f}$.

3 Lesky (1971) 914.

4 Zum Einfluss Alexanders des Großen auf die Ausgestaltung der Dionysos-Figur vgl. Stegemann (1930) 177 - 182; Adrados (2003). 
vergangenen eintausend Jahre dichterischen Schaffens in Griechenland zieht. Im selben Ausmaß, wie er auf das literarische Erbe zurückgreift, bietet er gleichwohl mindestens ebenso viele schöpferische Innovationen im metrischen und lexikalischen wie auch im stilistischen und inhaltlichen Bereich. Der sog. nonnianische Hexameter, eine Weiterentwicklung der kallimacheischen Gesetzmäßigkeiten, ist gekennzeichnet durch seine formale Strenge sowie seinen Schematismus und wird von einer ganzen Reihe spätantiker Poeten übernommen und gepflegt. ${ }^{5}$ Die sog. nonnianische Schule, Dichter des 3. bis 6. Jhs. n. Chr., weisen allesamt Ähnlichkeiten in Metrik, Lexik und Stilistik auf und pflegen die Konventionen spätantiker Dichtung als eine öffentlich vorgetragene „highbrow poetry“ “ ${ }^{6}$

Der Begriff nonnianische Schule relativiert sich insofern, als Lebenszeit und Wirkungsort dieser Literaten nicht immer in unmittelbarer Verbindung zueinander stehen. Viel eher als ein Initiator einer tatsächlichen Schule muss Nonnos daher aufgrund des Umfangs und des allumfassenden dichterischen Anspruchs als Schnittpunkt des dichterischen Schaffens der Spätantike gesehen werden.

Über den Dichter Nonnos selbst sind nur spärliche Informationen bekannt, lediglich sein Herkunftsort Panopolis, heute Akhmim in der oberägyptischen Thebais, darf als sicher gelten. ${ }^{7}$ Weder zu seiner Biographie noch zu seinem kulturellen Umfeld oder den Umständen seiner literarischen Tätigkeit sind Quellen bekannt, auch seine Lebenszeit ist nicht eindeutig festzumachen. Als terminus post quem werden Autoren des 4. und 5. Jhs. n. Chr. herangezogen, von denen Nonnos mutmaßlich oder nachweislich Kenntnis hatte. ${ }^{8}$

5 Für eine aktuelle Forschungsgeschichte zu Nonnos siehe Chuvin (2016). - Zu Nonnos' Hexameter und seinen Gesetzmäßigkeiten vgl. Golega (1930) 8-28; Wifstrand (1933); Keydell (1959) 35*-42*; Cameron Alan (1965) 482f.; Lindsay (1965) 365; Vian (1986) 339f.; Tissoni (1998) 26-29; Vian (1976) L-LV; Agosti (2004a); Greco (2004) 40-44; Hernández de la Fuente (2008) 29, 37 -39; Miguélez Cavero (2008) 106 - 114; Franchi (2013) 209-219; zum spätantiken Hexameter vgl. Agosti u. Gonnelli (1995).

6 Agosti (2011 - 2012) 233. - Triphiodor (3. Jh. n. Chr.), Pamprepios von Panopolis (zweite Hälfte des 5. Jhs. n. Chr.), Musaios (um 500 n. Chr.), Christodoros von Koptos (Anfang 6. Jh. n. Chr.), Kolluthos von Lykopolis (Anfang 6. Jh. n. Chr.), Paulos Silentiarios (6. Jh. n. Chr.) und Johannes von Gaza (6. Jh. n. Chr.) sind die wichtigsten Vertreter; zu Triphiodor vgl. Cameron Alan (1970) 478 - 482, Dubielzig (1996) und Miguélez Cavero (2013a); zu Pamprepios siehe Gerstinger (1928) und Livrea (1979); zu Musaios vgl. Accorinti (2012). - Zur Charakterisierung der spätantiken griechischen Dichtung und zu literarischen Vorgängern und Erben des Nonnos siehe auch Lindsay (1965) 362; Cameron Alan (1970) 4 - 6; Braden (1974) 68 -85; Vian (1980); Trypanis (1981) 395 - 407; Vian (1986); Dihle (1989) 611-617; Charlet (1997); Gigli Piccardi (2003) 7 -13; Hernández de la Fuente (2008) 227 - 237; Miguélez Cavero (2008) 12 - 15, 25 - 33; Agosti (2009); Agosti (2012). - Zur Praxis des öffentlichen Vortrags vgl. Agosti (2006); Agosti (2008a) 206f.

7 Zum Namen Nonnos vgl. Bonner (1954) 15-17; Dostálová-Jeništová (1955).

8 Der lateinische Dichter griechisch-ägyptischer Herkunft Claudius Claudianus (etwa 370 bis nach 404/405 n. Chr.), der eine Gigantomachie in griechischer Sprache verfasste - vgl. Abel-Wilmanns (1977) 25; Boscarino (1997); Livrea (2000a); Hernández de la Fuente (2001a) 19; Espinar Ojeda (2002) 3; Kyros von Panopolis, ein Zeitgenosse des Nonnos und Verfasser von Epigrammen in der Anthologia 
Bisweilen wird auch Beirut/Berytos, der Schauplatz des Beroe-Mythos und spätantikes Zentrum der römischen Rechtslehre, das ab 449/450 n. Chr. offiziellen Status einer Metropolis erhält, als Begründung für die Datierung des Nonnos in die zweite Hälfte des 5. Jhs. n. Chr. herangezogen. ${ }^{9}$ Die herausragende Rolle, welche die Stadt in den Dionysiaka einnimmt, veranlasste überdies dazu, für Nonnos einen Aufenthalt in dieser Stadt oder sogar ein Rechtsstudium ebendort anzunehmen; beides ist als bloße Hypothese $\mathrm{zu}$ werten. ${ }^{10}$ Als terminus ante quem dient Agathias von Myrina (6. Jh. n. Chr.), der in seinen Historien den Ägypter Nonnos und sein Werk, die Dionysiaka, namentlich nennt und ihn unter die „modernen Dichter“ rechnet (Agath. 4,23,5): ${ }^{11}$

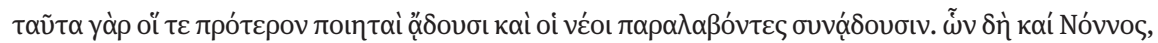

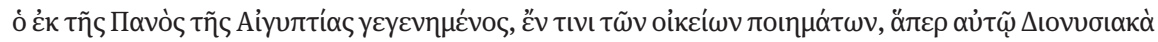
غ̇ं $\omega v o ́ \mu \alpha \sigma \tau \alpha \mathrm{L}[. .$.$] .$

Denn so singen die Dichter aus früheren Zeiten und die Neutöner haben das aufgenommen und singen in derselben Art. Einer von ihnen ist auch Nonnos, der aus Panopolis in Ägypten stammt, in einem seiner von ihm verfassten Gedichte, das er die Dionysiaka genannt hat [...].

Hinweise auf Nonnos' Herkunft und literarisches Schaffen finden sich auch in der Sammlung der Anthologia Palatina. Im Epigramm AP 9,198 wird darauf Bezug genommen, dass auch er, ähnlich wie Claudian, eine Gigantomachie verfasst habe: ${ }^{12}$

Palatina - vgl. Lindsay (1965) 361; Hernández de la Fuente (2001a) 19; Shorrock (2001) 144-146, 162 - 164; Hernández de la Fuente (2008) 27; van der Horst (2012); Gregor von Nazianz, der berühmte Kirchenlehrer des 4. Jhs. n. Chr. - vgl. Collart (1930) 10-13; Stegemann (1930) 207-209; AbelWilmanns (1977) 20 f.; zu Gregor und Nonnos vgl. D’Ippolito (1994); Simelidis (2009) 60 Anm. 138 mit weiteren Literaturangaben; Kyrill von Alexandria (etwa 375/380-444 n. Chr.), Autor epischer und epigrammatischer Dichtungen sowie eines Kommentars zum Johannes-Evangelium (425-428 n. Chr.), dessen sich Nonnos zur Abfassung seines zweiten Werks, der metrischen Paraphrase des JohannesEvangeliums, bediente - vgl. Gigli Piccardi (2003) $33 \mathrm{f}$.

9 Vgl. Stegemann (1930) 207; Hernández de la Fuente (2001a) 19; Espinar Ojeda (2002) 1f.; Bajoni (2003); Gigli Piccardi (2003) 35-37; Hernández de la Fuente (2008) 27. - Einen weiteren Datierungsansatz für Nonnos sah die Forschung auch in der Erwähnung der Blemmyer in Nonn. D. 17,385397. Dabei handelt es sich um ein am rechten Nilufer ansässiges Volk, das wiederholt in der Thebais einfiel; vgl. Dostálová-Jeništova (1956); Abel-Wilmanns (1977) 22f.; Gigli Piccardi (2003) 38-41; Barthel (2014). Pierre Chuvin zog außerdem die Blemmyer als Indiz für Nonnos' Neigung zum Heidentum heran, vgl. Chuvin (1991) 165 f., $278 \mathrm{f}$.

10 Vgl. Keydell (1936) 905, 915; Haidacher (1949) 102; Bonner (1954) 17; Trypanis (1981) 392; Liebeschuetz (1996) 86; Hernández de la Fuente (2001a) 20; Espinar Ojeda (2002) 2; Hernández de la Fuente (2008) 28; Lasek (2009) 67. - Zur Rechtsschule von Beirut/Berytos vgl. Liebeschuetz (2001a) 1030f., 1038f.; Jones Hall (2004) 195 - 213; Cribiore (2007a) 54, 57 f.

11 Text: Keydell (1967) 152; zum Zeugnis des Agathias vgl. Collart (1930) 4; Keydell (1936) 904; Lindsay (1965) 361; String (1966) 115-122; Abel-Wilmanns (1977) 10f.; Espinar Ojeda (2002) 3; Gigli Piccardi (2003) 33; García-Gasco (2007) 16; Hernández de la Fuente (2008) 27. - Zur Frage der Neuheit nonnianischen Dichtens vgl. Miguélez Cavero (2013a).

12 Text: Beckby (1965) Bd. 3, 122; Übersetzung: Ebener (1981) Bd. 2, 312. Zum Epigramm vgl. Keydell (1959) 9^; Abel-Wilmanns (1977) 9 f.; Livrea (1989) 32 f.; Hernández de la Fuente (2001a) 19; GarcíaGasco (2007) 16; Hernández de la Fuente (2008) 27 f. 


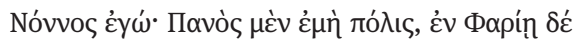

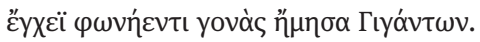

Nonnos heiße ich, stamme aus Panstadt. In Pharia mähte ich mit dem klingenden Schwert die Giganten scharenweis nieder.

Ob es sich bei dieser Gigantomachie um ein eigenständiges Werk oder etwa um die Dionysiaka selbst handelt, die als Abschluss der 48 Bücher Dionysos’ Kampf gegen die Giganten bieten, kann nicht mit Gewissheit entschieden werden. Die Nennung der Insel Pharos bildet den einzigen Anhaltspunkt für die These, Nonnos habe sich wie so viele seiner Zeitgenossen zu Studien- oder Erwerbszwecken im damaligen kulturellen und literarischen Schmelztiegel Alexandria aufgehalten.

Nonnos, dessen literarische Akme im Allgemeinen in die Mitte des 5. Jhs. n. Chr. gesetzt wird und der als Verfasser eines heidnischen und eines christlichen Werkes bekannt ist, ${ }^{13}$ gab der Gelehrtenwelt, namentlich Enrico Livrea, den Anlass zur Theorie, wonach es sich bei Nonnos von Panopolis um den gleichnamigen Bischof von Edessa handle (449-451 n. Chr.). ${ }^{14}$ Die Unklarheiten bei Identität und Datierung des Autors sowie beim Verhältnis zwischen Dionysiaka und Paraphrase lösten in der frühen Nonnos-Forschung eine Quaestio Nonniana aus, die, vergleichbar mit der homerischen Frage, ${ }^{15}$ die chronologische Relation zwischen den heidnischen Dionysiaka und der christlichen Paraphrase sowie die Konfessionszugehörigkeit des Autors untersuchte. Die zu Beginn der Forschung angenommene Konversion des Nonnos vom Heiden- zum Christentum und die Spätdatierung der Paraphrase, die überdies als Zeichen der reumütigen Umkehr eines treulosen Ungläubigen aufgefasst wurde, ${ }^{16}$ wurden jedoch bald zugunsten einer gemeinsamen christlichen Autorschaft beider Werke aufgegeben. ${ }^{17}$ Der gegenwärtige status quo geht davon aus, dass die Paraphrase

13 Arthur Ludwich (1909) VIII-X datiert die Dionysiaka zwischen 390 und 405 n. Chr., Keydell (1936) 902 zwischen 397 und 470 n. Chr., Friedländer (1912a) 440 - 490 n. Chr., Vian (1976) XVII, Braden (1978) 71 und Chuvin (1991) 7 f. jeweils zwischen 450 und 470 n. Chr., Rouse (1940) VII um 500 n. Chr.; vgl. Stegemann (1930) 206-209 mit einer Zusammenfassung der Datierungsvorschläge der frühen Nonnos-Forschung; zur Datierungsfrage siehe auch Golega (1930) 88 - 115; Lind (1934); Bury (1958) Bd. 2, 431 f. Anm. 4; Abel-Wilmanns (1977) 18 - 28; Cameron Alan (1982) 235 - 239; Baldwin (1986); Dihle (1989) 609; Manterola u. Pinkler (1995) 14f.; Tissoni (1998) 11-13; Garcia-Gasco (2007) 16; Hernández de la Fuente (2008) 27; Accorinti (2013) 1108-1110.

14 Vgl. Livrea (1987); Livrea (1989) 23 - 30; Livrea (2003); Gigli Piccardi (2003) 34; Hernández de la Fuente (2008) 31; gegen die Theorie Livreas: Cameron Alan (2000); vgl. auch Accorinti (2013) $1110 \mathrm{f}$. 15 Vgl. Abel-Wilmanns (1977) 14-18; Chuvin (1986) 338; Livrea (2000) 39-76; Hernández de la Fuente (2001a) 23; Gigli Piccardi (2003) 45-60; García-Gasco (2007) 15; Hernández de la Fuente (2008) 29-32; García-Gasco (2010) 7; Accorinti (2013) 1111f.

16 Vgl. Bury (1889) Bd. 1, 317 f.; Collart (1930) 13-15; Bogner (1934) 333; Keydell (1936) 915 f.; Cameron Alan (1965) 476; Bury (1958) Bd. 2, 434; Trypanis (1981) 392; Cameron Alan (2007) 36; Hernández de la Fuente (2008) 57.

17 Vgl. Golega (1930) 87 f., 143 f.; Dostálová-Jeništová (1955) 108; Dostálová-Jeništová (1958); Cameron Alan (2000) 181; Agosti (2001) 96 f.; Chuvin (2009a) 474; Cameron Alan (2011) 700f.; Whitby (2013) 218; Agosti (2014a) 297 f. - Alan Cameron geht in früheren Arbeiten davon aus, dass Nonnos 
ein Frühwerk sei, dem die Dionysiaka chronologisch nachgestellt werden müssten oder aber, dass eine zeitgleiche Abfassung beider Werke in Erwägung zu ziehen sei. ${ }^{18}$ In der neueren und neuesten Nonnos-Forschung wird das Hauptinteresse auf das kulturelle und soziale Umfeld der nonnianischen Dichtungen gelegt; ${ }^{19}$ die Spätantike wird nicht mehr als Periode des Niedergangs und Verfalls, sondern der Kontinuität, Dynamik und Multikulturalität gesehen. ${ }^{20}$ Der Dichter schafft seine beiden Werke in einer ungemein vielfältigen Gesellschaft, die sich durch äußerste Heterogenität im Bereich der religiösen und kultischen Ordnung ausweist. Heiden- und Christentum existieren über Jahrhunderte hinweg nicht bloß nebeneinander, sondern beeinflussen sich in hohem Maße gegenseitig. Neben der Langlebigkeit vieler heidnischer Kulte erfolgt die Annäherung von Christen- und Heidentum durch die Aufnahme philosophischen Gedankenguts durch die Christen, eine geistesgeschichtliche Fusion, welche im heidnische und jüdisch-christliche Traditionen verbindenden Neuplatonismus gipfelt. ${ }^{21}$ Die monotheistischen Züge des Neuplatonismus fügen sich ihrerseits in das üblicherweise als polytheistisch eingestufte Heidentum. ${ }^{22}$ Außerdem bleibt heidnische Bildung insbesondere die Kenntnis der Mythologie, animiert durch die Homer-Lektüre, sowie die rhetorische Schulung - auch für die Christen der Spätantike der Zugangscode zu führenden Positionen säkularer und kirchlicher Ämter.

Ein besonderes Spannungsfeld zwischen Heiden und Christen ist im spätantiken Ägypten zu beobachten, das nicht nur als das Mutterland des Mönchtums gilt, sondern

Heide war, vgl. Cameron Alan (1970) 192 Anm. 2. - Im Gegensatz zu den Ergebnissen der modernen Nonnos-Forschung wird die Konversions-These gelegentlich immer noch vertreten, vgl. Demandt (2007) 510.

18 Vgl. Beatrice (2007); Cameron Alan (2011) 700. - Um die Abfassungszeit der Paraphrase zu bestimmen, werden neben Einflüssen patristischer Autoren wie Kyrill von Alexandria (ca. 375/380 - 444 n. Chr.) auch die Konzilien herangezogen, als terminus post quem das Konzil von Ephesos $431 \mathrm{n}$. Chr. mit seinen mariologischen Fragestellungen sowie das Konzil von Chalkedon 451 n. Chr. mit der Problematik der Christologie und Trinitätslehre; vgl. Golega (1930) $111 \mathrm{f}$.; Stegemann (1930) 208; Thraede (1962); Trypanis (1981) 392.

19 Vgl. Bowersock (1990) 1-40; vgl. dazu Kap. 8.2.

20 Als für die moderne Spätantike-Forschung wegbereitend dürfen die Arbeiten von Peter Brown gewertet werden (siehe die Bibliographie); zur kulturellen Situation des griechischen Ostens in der Spätantike vgl. den Überblick in Jones Ch. (2014) 126-143; zur Entwicklung der Spätantike-Forschung vgl. Mazzarino (1951) 7 -31; Fuhrmann (1967); Herzog (1987); Cameron Averil (2004); Liebeschuetz (2004); Athanassiadi (2006); Formisano (2007); James (2008); Giardina (2013). - Zur Verfallstheorie vgl. Dodds (1985); Lot (1996) 151-170; zur Kritik der Verfallstheorie vgl. Mazzarino (1961) 184-202.

21 Vgl. Hernández de la Fuente (2008) 4, 6f.; Cameron Averil (2012) 134-136; Hernández de la Fuente (2014a).

22 Wissenschaftliche Arbeiten vor allem der vergangenen zehn Jahre zeigen auf, dass dieser pagane Monotheismus ein wesentliches Merkmal der spätantiken Gesellschaft darstellt; vgl. Fowden (1993a) sowie die Sammelbände Mitchell u. Van Nuffelen (2009) und Mitchell u. Van Nuffelen (2010) sowie Athanassiadi u. Frede (2002), darin besonders Frede (2002) und West (2002); Edwards (2004); Liebeschuetz (2006) 984-1008; Kahlos (2007) 145-147; Hernández de la Fuente (2008) 7-9, 13; Cerutti (2010); Eich (2010). 
bis zum Ausgang der Spätantike auch Bastion für das Heidentum bleibt. ${ }^{23}$ Eklektizismus und Synkretismus sowie die gegenseitige Übernahme religiöser Ausdrucksformen ermöglichen hier kulturelle Synergien, die den Grundstein für die zeitgenössische Dichtung legen, die auf die klassisch griechische Literatur rekurriert, ererbte Motive und Konventionen transformiert und literarische Traditionen für ihre eigene Zeit nutzbar macht. ${ }^{24}$ Ein Zentrum heidnischer Dichtung und hellenistischen Selbstverständnisses ist Nonnos’ Heimatstadt Panopolis, eine Bildungsstätte, die nachweislich zahlreiche Gelehrte und Literaten anzog, darunter etwa die Dichter Pamprepios und Kyros oder den Philosophen Horapollon. ${ }^{25}$ Das kulturelle Milieu, das auf der gegenseitigen Einflussnahme des aufstrebenden Christentums und den beständig florierenden paganen Traditionen fußt, wird von offiziellen Bildungseinrichtungen getragen. ${ }^{26}$ Panopolis in Oberägypten ist nur ein Beispiel für die zahlreichen Bildungszentren im griechischsprachigen östlichen Mittelmeerraum; die neuplatonische Schule in Athen, die Philosophie- und Rhetoriklehrstühle in Konstantinopel, Antiochia, Alexandria und Gaza sowie die Rechtsschule in Beirut legen Zeugnis ab von der Bedeutung der Ausbildung in den heidnischen Wissenschaften. ${ }^{27}$ Wenngleich keinerlei gesicherte Informationen zu Nonnos' Person existieren, müssen dennoch ebendiese kulturellen Rahmenbedingungen als Voraussetzungen für sein Literaturschaffen angenommen werden. Die sowohl in den Dionysiaka als auch in der Paraphrase des Johannes-Evangeliums zu findenden heidnischen und christlichen Motive und Sprachelemente zeugen von der spätantiken Vorliebe für synkretistische Tendenzen, ${ }^{28}$ die alte Frage nach der Konfession des Autors erscheint vor diesem Hintergrund folglich ebenso obsolet wie der in der Forschung über lange Zeit propagierte unüberbrückbare Antagonismus zwischen Heiden- und Christentum.

23 Vgl. Schulze (1971); Brown (1978) 91f.; Bowersock (1990) 55-69; Bowman (1996) 121-164; Török (2005) 51-111; Hernández de la Fuente (2008) 13-19.

24 Zur literarischen Produktion im spätantiken Ägypten und zur Situation der Dichter vgl. Cameron Alan (1965); Cameron Alan (2004); Miguélez Cavero (2008).

25 Vgl. Cameron Alan (1965) 471; Cameron Alan (1982); Chuvin (1986) 388-390; Bagnall (1993) 103 f., 109; Bowman (1996) 162f., 232; Liebeschuetz (1996) 83; Frankfurter (1998) 222 - 224; Cribiore (2001) 200; Hernández de la Fuente (2001a) 18; Egberts u. a. (2002) VII-XI; Espinar Ojeda (2002) 2 f.; Cameron Alan (2007) 34; García-Gasco (2007) 16; Hernández de la Fuente (2008) 16 -18; López (2013) 21.

26 Allein für Panopolis sind drei Professuren für das Ende des 4. Jhs. n. Chr. überliefert, vgl. Hernández de la Fuente (2008) 16; zu Panopolis als Ort mit Bibliothek und als intellektuellem Zentrum vgl. Bagnall (1993) 103 f., 109; zu Panopolis allgemein siehe Van Rengen (2013).

27 Vgl. den Überblick in Demandt (2007) 481 - 488 und Demandt (2013); außerdem Liebeschuetz (1992) 26; zu Alexandria vgl. Bowersock (1996); Bowman (1996) 203-232; MacCoull (2007); Benaissa (2012) 535-538; zum Heidentum im spätantiken Alexandria und zu dessen Verhältnis zu anderen Religionen vgl. Haas (1997) 128-172.

28 Vgl. Brown (1978) 97. Zum Synkretismus im spätantiken Kleinasien siehe Lozano (2010), zu Ägypten vgl. Krause u. Hoheisel (2001) 15 f., und zum synkretistischen Weltbild in den Dionysiaka vgl. Accorinti (1995). 
Mit den 48 Büchern und über 21.000 Hexametern der Dionysiaka, einer Biographie des Weingottes Dionysos von seinen Vorfahren bis zu seiner Apotheose im Götterhimmel, bewegt sich der Autor in ebendiesem vielschichtigen kulturellen und literarischen Umfeld. ${ }^{29}$ Die äußerst breit angelegte Erzählung um Dionysos’ Leben und Wirken auf Erden und die Verbreitung seines Kultes ist durchsetzt von zahllosen exkursartigen Passagen und Digressionen, die jede für sich genommen einen ausgewählten Aspekt des dionysischen Prinzips thematisieren und sich so in die übergeordnete Erzählung um Dionysos fügen. Das neue dionysische Epos distanziert sich von den epischen Traditionen, arbeitet aber gleichzeitig mit sprachlichen Versatzstücken und Motiven homerischer Dichtung, entwickelt sie weiter und fügt Elemente anderer Genera hinzu - Bukolik und Hymnos gehören ebenso zum nonnianischen Repertoire wie Rhetorik und Epigrammatik. ${ }^{30}$ Neben der enzyklopädischen Vielfalt an Mythen die Dionysiaka werden als ,una gran summa mythologica“31 eingestuft - wird vor allem der überbordende, barock anmutende Stil als Charakteristikum der Dionysiaka angesehen. ${ }^{32}$ Der Schauplatz ist die ganze Welt, der Olymp mit inbegriffen, räumliche wie sprachliche Grenzen werden aufgehoben, gewagte sprachliche Neologismen und Eigenkreationen des Dichters kumulieren in ein rauschhaftes, dionysisches Crescendo. ${ }^{33}$ Aktuelle Forschungstendenzen, denen auch das vorliegende Buch verpflichtet ist, sehen gerade in diesem scheinbaren Chaos der Komposition sowie in der Technik der Anpassung von Sprache und Stilistik an das Thema das wesentliche Merkmal nonnianischen Dichtens. ${ }^{34}$

\subsection{Die Dionysiaka und die Ampelos-Episode}

Trotz der Heterogenität in der Komposition bildet das dionysische Prinzip die Leitlinie, die auch einen wesentlichen kompositorischen Aspekt der Ampelos-Episode aus-

29 Zur handschriftlichen Überlieferung der Dionysiaka siehe Marcellus (1856) XII-XXXVIII; Maas (1923) Nr. XX (167 f.); Keydell (1936) 916f.; Keydell (1959) 11*-27*; Schmid u. Stählin (1918/61924) 970f.; Manterola u. Pinkler (1995) 41f.; Tissoni (1998) 36-39, 44-62; Hernández de la Fuente (2001a) 28-31; Espinar Ojeda (2002) 1; Lind in Rouse (1940) XX-XXV; Gigli Piccardi (2003) 31f.; Hernández de la Fuente (2006) 148-154; Hernández de la Fuente (2008) 26.

30 Vgl. Agosti (2001) 95: „Nonno ha programmaticamente inteso fare un'epica nuova, che rompesse con il passato.“

31 Cuartero i Iborra (2003) 177.

32 Zur Charakterisierung des nonnianischen Stil als barock vgl. Wifstrand (1933) 81f.; D’Ippolito (1964) 44 - 57; Hernández de la Fuente (2008) 40 - 57; van Opstall (2013). - Zum nonnianischen Stil allgemein siehe Riemschneider (1957); Agosti (1995); Accorinti (2009); Accorinti (2013) 1116f. - Zum Terminus barock in der Spätantike siehe auch Picard (1990) 266-273.

33 Vgl. Dihle (1989) 610; Hernández de la Fuente (2008) 54; Lesky (1971) 914: „Die Dionysiaka sind bei allem Reichtum motivischer und formaler Voraussetzungen doch ein Werk eigenen Gepräges. Sie sind es durch den Zug dionysisch-rauschhafter Erregung, der durch das Ganze geht.“

34 Vgl. Hernández de la Fuente (2008) 7, 23, 34: „sincretismo subjetivo“ und „nueva subjetividad“; siehe auch Hernández de la Fuente (2008) 35-37; Brioso Sánchez (1994-1995). 
macht. Das Epos präsentiert sich in den großen Linien als biographische Lebensgeschichte des Weingottes Dionysos und schildert dessen Leben und Triumphzug durch die Welt sowie die flächendeckende Etablierung seines Kultes. ${ }^{35}$ Die drei Erzählblöcke lassen sich wie folgt gliedern: Die Bücher 1-12 liefern die Vorgeschichte des späteren Gottes Dionysos und erzählen Mythen rund um seine Familie, seine Geburt, Kindheit und Jugend. Den größten Umfang nimmt der zweite Block ein, der in 28 Büchern Dionysos' Taten wiedergibt, insbesondere den Feldzug gegen die Inder (Nonn. D. 13 40). In Block drei wendet sich der Gott nach der erfolgreichen Verbreitung seines Kultes im Osten nun dem europäischen Kontinent zu und zieht nach Griechenland (Nonn. D. 41-48); die äußerst knapp geschilderte Apotheose beschließt das Epos.

Der erste Block der Erzählung setzt „ab ovo ovi““36 mit der mythischen Urgeschichte des Gottes ein, die - an das Proömion mit dem Anruf der bakchischen Musen und der Deklarierung der Dionysiaka als proteische Dichtung ${ }^{37}$ anschließend - die Vorgeschichte um Europa, Kadmos und Typhon schildert (Nonn. D. 1-2). ${ }^{38}$ Auf der Suche nach der von Zeus entführten phönizischen Königstochter, seiner Schwester Europa, steht Kadmos dem Göttervater höchstpersönlich im Kampf gegen den Usurpator Typhon bei; nur mit vereinten Kräften und durch Anwendung einer Finte - die Bezauberung Typhons durch bukolische Flötenmusik - gelingt es ihnen, das chimärenartige Mischwesen zu überwältigen und die göttliche Ordnung mit Zeus an der Spitze wiederherzustellen. Die folgenden beiden Bücher (Nonn. D. 3-4) schildern Kadmos' Begegnung mit seiner Braut Harmonia auf der Insel Samothrake, die Schifffahrt der beiden nach Griechenland, die Gründung Thebens und das Schicksal ihrer Kinder, Autonoe, Ino, Agaue, Polydoros und Semele. Was im Zusammenhang mit der Familie des Kadmos bereits angedeutet wird, das Wirken dionysischer Kräfte in der Welt, wird in den Büchern 6-8 weiter ausgeführt: Auf das Leben und den tragischen Tod des Zagreus, des ersten Dionysos, folgen Weltenbrand und Sintflut, zwei Naturkatastrophen, die einen klaren Einschnitt in der Chronologie des Epos schaffen und die Vorgeschichte beenden. Die Sintflut beschließt eine alte Göttergeneration und gleichzeitig die Vorgeschichte, die „Archäologie“399, das „Antiguo Testamento“40 der Dionysiaka, unmittelbar danach beginnt das neue, dionysische Zeitalter, das von Buch 7 und Zeus' fruchtbarer Verbindung mit Semele seinen Ausgang nimmt ${ }^{41}$ und einen

35 Für einen Überblick über den Inhalt der Dionysiaka siehe auch Chamberlayne (1916) 43-45; D’Ippolito (1964) 58 - 68; Manterola u. Pinkler (1995) 19 - 38; Shorrock (2005) 375 f.; Frendo (2006) 283 f.; Gigli Piccardi (2003) 27 -30; Miguélez Cavero (2008) 19-21.

36 Schmid u. Stählin (1980/61924) 967.

37 Zum Proömion vgl. Schmitz (2005) 202 -208; Frendo (2006) 278 - 280; Hernández de la Fuente (2008) 40-52; Bannert (2008); Accorinti (2013) 1116.

38 Vgl. D'Ippolito (1964) 191-214. Zur Typhonomachie siehe Braden (1974); Schmiel (1992); Cuartero i Iborra (2003) 180 - 184; zu Kadmos siehe Cuartero i Iborra (2003) 184-186; Aringer (2012). 39 Vian (1976) XXIII: „Les ch. 1-12 forment un tout clair et équilibré. Six chants de préliminaires constituent une ,Archéologie“ dionysiaque.“

40 Hernández de la Fuente (2008) 213.

41 Vgl. D’Ippolito (1964) 228-240. 
ersten Höhepunkt in der Schenkelgeburt und in den Kindheitserzählungen um Dionysos erfährt (Nonn. D. 8). Nach der tragischen Geschichte um Ino, Athamas und deren Kinder (Nonn. D. 9-10) ${ }^{42}$ setzt die Ampelos-Episode der ausführlichen Jugendgeschichte des Gottes ein Ende (Nonn. D. 10 -12).

Im zweiten und umfangreichsten Block alterniert die lange Erzählung um Dionysos' Kriegszug gegen die Inder mit einer Reihe von Episoden, die sich außerhalb des Kampfkontexts bewegen. ${ }^{43}$ Die Bücher 13 und 14 bieten mit dem Katalog der dionysischen Truppen eine erste Konfrontation der beiden Heere, die Anwendung dionysischer Kampfmethoden - die Inder werden durch das Trinken aus einem Wein führenden Fluss kampfunfähig gemacht - bringt einen Aufschub des Kampfes. Die Bücher 15 und 16 blenden das Kriegsgeschehen vorübergehend aus und erzählen die bukolisch inspirierte Geschichte von Hymnos und Nikaia; in den Büchern 17 sowie 18 und 19 erfolgt sodann die gastliche Aufnahme des Dionysos durch den Hirten Brongos und den syrischen König Staphylos. In beiden Erzählungen (mit den sprechenden Namen der Protagonisten) findet sich Dionysisches: Brongos verkostet das neue Getränk, den Wein, und Staphylos und seine Familie - seine Gattin Methe, sein Sohn Botrys und der alte Pithos - werden Teil des dionysischen Kultes. In den Büchern 20 und 21 sieht sich der Gott mit seinem Gegner Lykurg konfrontiert, der jedoch schließlich von der in einen Weinstock verwandelten Ambrosia überwältigt wird. Nach dem ersten Höhepunkt der Kämpfe, der Schlacht am indischen Fluss Hydaspes (Nonn. D. 22-24), setzt mit dem erneuten Musenanruf, der Berufung auf das große dichterische Vorbild Homer und der Schildbeschreibung (Nonn. D. 25) ein Neubeginn des Krieges ein, der mit einer Heeresversammlung und einem Götterrat beginnt (Nonn. D. 26-28). Der in den Büchern 29 und 30 geschilderte Kampf auf Menschen- und Götterebene wird in der Folge mehrmals unterbrochen: Der von Hera geschickte Wahnsinn macht Dionysos vorübergehend kampfunfähig (Nonn. D. 31-32), die Geschichte um die unglückliche Liebe des Inders Morrheus zur Bakche Chalkomedeia dient weniger der Überbrückung der Kampfpause als vielmehr der Beleuchtung weiterer Aspekte dionysischer Wirkkraft (Nonn. D. 33-35). Der in Buch 36 angekündigte kriegsentscheidende Zweikampf zwischen dem mittlerweile vom Wahnsinn genesenen Dionysos und Deriades, dem Heerführer der Inder, wird bis zu den Büchern 39 und 40 aufgeschoben; dazwischen werden die Leichenspiele für Opheltes sowie der Mythos um Phaethon geschildert. Das Ziel des zweiten Erzählblocks ist mit dem Kriegsende und dem Sieg des Dionysos über die Inder in Buch 40 erreicht, nachdem sich der Gott in seinen kriegerischen Unternehmungen bewährt hat und nun bereit ist, seine göttlichen Kräfte auch in Friedenszeiten einzusetzen. Dionysos zieht gen Westen, stattet Tyros und dem Stadtgott Herakles-Astrochiton (Nonn. D. 40) ebenso einen Besuch ab wie Beirut, wo er um die Gunst der Aphrodite-Tochter Beroe in einen Konkurrenzkampf mit Poseidon tritt

42 Vgl. D’Ippolito (1964) 240 - 252.

43 Zum Kriegszug des Dionysos gegen die Inder und zu dessen kompositorischer Funktion vgl. Vian (1994a). 
(Nonn. D. 41-43). Dionysos gelingt es, nach Überwindung weiterer Gegner - Pentheus (Nonn. D. 44-46) und Hera (Nonn. D. 47) - seinen Kult auch in Griechenland zu etablieren und den Athener Ikarios die Kunst des Weinbaus zu lehren (Nonn. D. 47). In Buch 48 bietet Nonnos eine breite Palette an dionysischen Themen und lotet seine Poetik im Zeichen des Weingottes mit der Gigantomachie, den Begegnungen mit Pallene und Aura, der Geburt des Iakchos, des dritten Dionysos, und mit Dionysos' Apotheose noch einmal aus.

Da sich die Ampelos-Episode am Ende des ersten Viertels der Dionysiaka an einem Knotenpunkt der Erzählung befindet, ${ }^{44}$ laufen viele Fäden in den Büchern 10-12 zusammen, finden sich zahlreiche Motive und Themen in früheren Büchern angekündigt und werden in späteren wiederholt oder modifiziert. Die Bücher 10 - 12 führen den narrativen Plan der Dionysiaka einen wesentlichen Schritt weiter, indem sie durch die Genese des Weines und die Ausstattung des Dionysos mit seinem Hauptattribut dem Epos nun dionysisches Gepräge verleihen. Die prominente Funktion, die die Ampelos-Episode im Epos-Ganzen einnimmt, rechtfertigt die detaillierte Analyse dieser Passage.

\subsection{Forschungsgeschichte zur Ampelos-Episode}

\section{Erste Eindrücke}

Die zentralen Monographien und Aufsätze zu Nonnos greifen einzelne Aspekte der Bücher 10 - 12 heraus und ziehen Rückschlüsse auf die Anlage des Gesamtepos, wobei die meisten frühen Forschungsansätze von einer negativen Tendenz gekennzeichnet sind. ${ }^{45}$ Ein durchaus positives Urteil über die Ampelos-Episode findet sich bereits im 1817 erschienenen Nonnos-Buch des St. Petersburger Gelehrten Sergej Semjonowitsch Ouwaroff, ${ }^{46}$ der zwei Punkte vorwegnimmt, die in der späteren Nonnos-Forschung Bedeutsamkeit erlangen werden: (1.) die Aufnahme verschiedener literarischer Genera sowie (2.) die Relevanz der zahlreichen Motivwiederholungen im Hinblick auf die

44 Für eine Inhaltsangabe zur Ampelos-Episode vgl. u. a. Dupuis (1847) 135 - 203; Chrétien (1985) 52 - 54, 81; Hernández de la Fuente (2008) 80 - 85; Mazza (2012) 100; Egetashvili (2013) 56-105, siehe auch die Nacherzählung des Mythos in Dalby (2005) 55-59.

45 Ähnlich wie die Ampelos-Episode wurden auch die Erzählungen über Aktaion, Nikaia, Aura und Pallene als bloße Zwischenspiele abgetan, vgl. String (1966) 6-17, bes. 14 über die Hymnos-NikaiaEpisode: „Wir haben an unserer Geschichte eine zusammenhängende Erzählung vermißt, fanden auch keineswegs statt dessen einzelne Szenen oder Situationen intensiver ausgeführt, vielmehr eigentlich nur lauter Einzelstücke, zu denen jeweils das Thema aufforderte, die aber auf einander wenig Rücksicht nahmen. Es fehlte das Ganze, aber auch das Einzelne macht den Eindruck, als sei es nicht aus der Situation heraus entstanden, sondern eher auf sie angewendet.“

46 Vgl. Ouwaroff (1817) 29 - 42. Zu Ouwaroff vgl. auch Aringer u. a. (2011) 1 - 3. - Ein Überblick über die frühe Forschung zur Komposition des gesamten Epos, im Wesentlichen der ersten Hälfte des 20. Jhs., findet sich in D’Ippolito (1964) 5-36; Abel-Wilmanns (1977) 45- 53. 
Gesamtkomposition des Epos. Den Einfluss der Literaturgattungen exemplifiziert Ouwaroff anhand der Rede des Dionysos an Ampelos, in der er epigrammatische Elemente enthalten sieht (Nonn. D. 10,196-216). Während er die Variante zur Genese des Weines am Ende der Ampelos-Episode nur als weitläufige Erzählung einstuft und nicht näher kommentiert, betrachtet er die Geschichte um Kalamos und Karpos und ihre Motivdoppelungen als charakteristisches Stilprinzip des Nonnos. ${ }^{47}$

Ähnlich wie auch in der Homer-Forschung wird in den frühen Arbeiten zu den Dionysiaka ein analytischer Ansatz gewählt, etwa von August Scheindler, der für eine nachträgliche Einfügung der Horen-Episode in die Bücher 11 und 12 plädiert (Nonn. $D$. 11,485-12,117), ${ }^{48}$ indem er vor allem an der ausführlichen Ekphrasis der Horen Anstoß nimmt, durch die die Nennung des eigentlichen Grundes für die Konsultation des Helios durch die Göttinnen der Jahreszeiten aufgeschoben werde, sowie an den beiden Trauerreden des Dionysos. Ungereimtheiten sieht Scheindler ferner im Mythos selbst begründet, wonach in der Schilderung in den Dionysiaka eine Verschränkung von zwei ursprünglich getrennten Mythen vorliege: In der Ur-Fassung seien es nicht die Horen, sondern die Moiren gewesen, die ,aus Erbarmen über Bacchos’ Trauer die Verwandlung des Ampelos und damit die Erfindung des Weines bewirken (Nonn. D. 12,139)“. ${ }^{99}$

In die Tradition der Analyse zu stellen ist auch Paul Collart, der als einer der Ersten eine Gesamtinterpretation der Dionysiaka vorlegt. Auch für ihn ist die Kalamos-KarposEpisode ein sekundärer Einschub, tatsächlich müsse auf Nonn. D. 11,350 der Vers Nonn. D. 11,484 folgen, zudem widerspreche der Vers Nonn. D. 11,358f. der Wiederaufnahme der Ampelos-Handlung in Nonn. D. 12,117 f. ${ }^{50}$ Für das 12. Buch konstatiert Collart ebenfalls einen Bruch in der Komposition, indem er dem Alternativmythos um die Genese des Weines eine logische Begründung abspricht. ${ }^{51}$

Viktor Stegemanns kosmologisch-astrologische Interpretation der Dionysiaka sieht die Komposition des Epos in Analogie zur Darstellung des kosmischen Jahres auf den Tafeln der Harmonia komponiert, wobei der Anzahl von 48 Büchern eine besondere Bedeutung zukomme:52 Dionysos durchschreite im Fortgang der Ereignisse die vier Jahreszeiten mit jeweils zwölf Tierkreiszeichen. Stegemanns These, wonach die Dionysiaka ausschließlich und durchgehend auf diese astrologische Linie hin ausgerichtet seien, fand Kritik durch Rudolf Keydell, der in seinem Aufsatz „Eine NonnosAnalyse“ aus dem Jahr 1932 eine knappe Stellungnahme zur Geschichte um Ampelos

47 Vgl. Ouwaroff (1817) 40: „Von dieser Seite sind überhaupt alle Sagen des Nonnos symbolisch.“

48 Vgl. Scheindler (1880) 34-37.

49 Scheindler (1880) 36.

50 Vgl. Collart (1930) 105-107. - Die Beschreibung des Ringkampfes zwischen Dionysos und Ampelos im 10. Buch wird ebenfalls als unvollendet eingestuft, vgl. Collart (1930) $99 \mathrm{f}$.

51 Vgl. Collart (1930) 108-110. - Die Inkonzinnität der Episode setze sich, so Collart, in einer zum Tod des Ampelos durch den Stier alternativen Version, dem Tod durch ein Raubtier, fort, welche noch in Ansätzen im friedlichen Umgang des Satyrn mit den Tieren des dionysischen Zugs zu erkennen sei; vgl. Collart (1930) 101-105; Shorrock (2001) 13-16.

52 Vgl. Stegemann (1930). 
liefert und die These Collarts, wonach die Ampelos-Geschichte bei Nonnos sich aus ursprünglich zwei unterschiedlichen Ampelos-Mythen zusammensetze, zurückweist und die ganze Episode als sekundäre Zutat einstuft. ${ }^{53}$ Die gesamte Partie zwischen dem Ende der ersten Klagerede bis zum Beginn der Rede der Atropos in Nonn. D. 12,138 müsse, so Keydell, als späterer Einschub gewertet werden. Keydell schließt sich seinen Vorgängern Scheindler und Collart an, wenn er die Kalamos-Karpos-Geschichte ebenso wie den Besuch der Horen bei Helios für eine sekundäre Zutat hält ${ }^{54}$ und sich für eine nachträglich vorgenommene Bucheinteilung ausspricht.

\section{Nonnianische Poetik und Komposition}

Ein Bruch mit der analytischen Interpretation und eine erste positive Würdigung erhalten die Dionysiaka durch Helmut Haidacher, der auf die Einflüsse unterschiedlicher literarischer Genera verweist: „Nicht nur das Epos, das ihm natürlich am nächsten lag, imitiert er, sondern auch pindarische Chorlyrik, Tragödie, Epigramm-, Hymnendichtung, Prosaroman und zeitgenössische Rhetorik sind ihm in gleicher Weise zur Ausschmückung seines Werkes willkommen. " ${ }^{55}$ Bezüglich der Ampelos-Episode verweist Haidacher auf den Bezug zu Achilleus Tatios und die Episode um Charikles und dessen Sturz von einem Pferd (Ach. Tat. 1,12-14); zudem sei in den Klagereden des Dionysos die rhetorische Tradition ebenso unverkennbar wie in den Reden Ates und Selenes. ${ }^{56}$ Haidacher hebt auch erstmals die Bedeutung der Ampelos-Episode für die Gesamtaussage des Epos hervor: „Mit der Erfindung des Weins, der ein neues Zeitalter einleitet, endet der erste große Abschnitt des Epos. “57

An den Einfluss der antiken Genera knüpft in der Folge Gennaro D’Ippolito an, der in seinen Studi Nonniani die Ampelos-Episode als Epyllion klassifiziert und eine Analyse des Aufbaus liefert. ${ }^{58}$ Er schreibt die Punkte, an denen Scheindler, Collart und Keydell Anstoß nahmen, dem spezifischen Charakter nonnianischer Dichtung zu; so sei es der Tradition der Epylliendichtung zuzurechnen, dass Nonnos bevorzugt mit

53 Vgl. Keydell (1932) 179-182 (491-494); vgl. auch Keydell (1931) 115f. (147f.). - Zur analytischen Interpretation der gesamten Dionysiaka siehe außerdem Keydell (1927); Krafft (1975); Vian (1980) $78 \mathrm{f}$.

54 Dafür spreche, so Keydell (1932) 180f. (492 f.), auch die allgemeine „Situation, die 11,312 besteht und die wir 12,138ff. wieder finden, dass nämlich Dionysos klagend vor der Leiche seines Freundes steht; vielmehr passt die Schilderung der Trauer nur, wenn schon einige Zeit seit Ampelos' Tode vergangen ist. Also auch dieses Stück ist eingeschoben." Keydell versucht innerhalb der langen Passage noch weiter zu differenzieren und unterschiedliche kompositorische Schichten herauszulösen.

55 Haidacher (1949) 148.

56 Vgl. Haidacher (1949) 96-100, 119-121.

57 Haidacher (1949) 121.

58 Vgl. D’Ippolito (1964) 131-149; vgl. Shorrock (2001) 16f.; zum Einfluss des Epyllions auf Nonnos siehe Hollis (2006). 
kontrastierenden Bildern arbeite. ${ }^{59}$ D'Ippolito sieht die kosmologische Partie mit dem Besuch der Horen bei Helios und die Kalamos-Karpos-Geschichte nicht als sekundäre Zutat innerhalb der Ampelos-Episode, ${ }^{60}$ sehr wohl jedoch - aufgrund der Inkompatibilität mit der zweiten Klagerede des Dionysos - die Prophezeiung über den Tod des Ampelos in Nonn. D. 11,83-98. ${ }^{61}$ Ähnlich wie Haidacher verweist auch D’Ippolito auf das motivische Vorbild Achilleus Tatios, stellt aber eine direkte Abhängigkeit vom Romanautor in Frage. Ebenso verhalte es sich mit den Parallelen zwischen Ampelos und einer ganzen Reihe von mythischen Vergleichsfiguren, deren direkte Quellen nicht zu ermitteln seien. ${ }^{62}$

Der strukturalistische Ansatz von Barbara Abel-Wilmanns sieht die bislang weitgehend negativ beurteilten Charakteristika der Dionysiaka - Episodenhaftigkeit, Wiederholung von Themen und biographisch-genealogische Tendenzen - ebenso als Elemente der Kohärenz wie die Einbeziehung unterschiedlicher Genera. ${ }^{63}$ Die in den Dionysiaka zugunsten einzelner, oft deskriptiver Partien zurückgetretene Handlung sei als typisch spätantik einzustufen. Auch Wolfgang Fauth wertet in seiner 1981 erschienenen Monographie Motivwiederholung und Kontrast als typisch nonnianische Kompositionsprinzipien, die auch in der Szene um Dionysos und die badenden Satyrn $\mathrm{zu}$ beobachten seien und sich im kontinuierlichen Hinarbeiten auf das narrative Ziel, die Schaffung der Weinrebe und die Ausstattung des Weingottes, zeigten: Die Genese von göttlichen Attributen erfahre in den Metamorphosen von Methe, Botrys und Pithos im 18. Buch eine Wiederaufnahme. ${ }^{64}$ Wichtige Beobachtungen zur Komposition der Dionysiaka, zur Technik der тоıкıía und zum Prinzip der kontrastiven Gegenüberstellung werden zum Ausgangspunkt für nachfolgende Forscher. ${ }^{65}$ So verweist etwa Thierry Duc auf die kompositorische Funktion von Wiederholung und variatio auf sämtlichen Ebenen der nonnianischen Sprache; wesentlich bei Nonnos sei nicht mehr der bloße Inhalt eines Mythos, sondern die Art und Weise seiner Präsentation. ${ }^{66}$ Auch Pierre Chuvin verweist auf die motivischen Parallelen der Ampelos-Episode zu anderen Teilen der Dionysiaka, indem er zwischen den Figuren Ampelos, Hymnos, Atymnios und Hymenaios Verbindungslinien zieht. ${ }^{67}$ Die bei Chuvin behandelte Kernthematik eines möglichen Bezugs der Dionysiaka auf die Zeit des Autors werde im

\footnotetext{
59 D’Ippolito (1964) 133: „Quindi un alternarsi di quadri sereni e di quadri tragici, una composizione a tableaux contrastanti, cara alla tradizione poetica dell'epillio.“

60 Vgl. D’Ippolito (1964) 137f., 146-149. Auch D’Ippolito weist hier die These Collarts zurück, wonach die Ampelos-Episode in den Dionysiaka aus zwei ursprünglich voneinander unabhängigen Mythen um Ampelos schöpft.

61 Vgl. D’Ippolito (1964) $138 \mathrm{f}$.

62 Vgl. D’Ippolito (1964) 140-145.

63 Vgl. Abel-Wilmanns (1977) 37-44, 139-182, 72-75, 83-93, 98-107.

64 Vgl. Fauth (1981) 136-139.

65 Vgl. Fauth (1981) $47-49,141 \mathrm{f}$.

66 Vgl. Duc (1990).

67 Vgl. Chuvin (1991) 58. - Zur Verankerung der Ampelos-Episode mittels des Kompositionselements Metamorphose vgl. Chuvin (2006) 250.
} 
Besonderen an den Tafeln der Harmonia greifbar, die allesamt eine Verbindung zu Kleinasien aufweisen. ${ }^{68}$

Auch Francis Vian, der Pionier der französischen Nonnos-Forschung, sieht die alternative Erzählung über die Entstehung des Weines, die für Keydell im Anschluss an

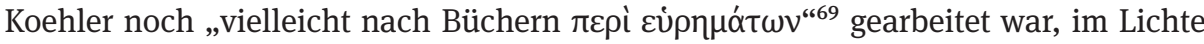
nonnianischer Erzähltechnik ${ }^{70}$ und zeigt durch Herausarbeiten struktureller Ähnlichkeiten und Wortwiederholungen, dass beide Versionen nach dem Variationsprinzip verschiedene thematische Schwerpunkte setzen und einander ergänzen. ${ }^{71}$ Hinsichtlich der Frage nach lateinischen Quellen zeigt Vian die motivischen Parallelen zwischen der Erfindung des Weines in den Dionysiaka des Nonnos und in Nemesians Bukolika auf, kann jedoch keine gesicherte Abhängigkeit feststellen, sondern nimmt eher eine beiden Autoren gemeinsame Quelle an. ${ }^{72}$

Eine Reihe von Arbeiten hebt die Motivwiederholungen und -variationen als wesentliche Aspekte nonnianischer Dichtung hervor, so etwa Daria Gigli Piccardi, die in ihrer Monographie über den Gebrauch von Metaphern bei Nonnos auch diejenigen zur Charakterisierung des jungen Satyrn abhandelt, ${ }^{73}$ oder Danièle Auger, die im Rahmen ihrer Zusammenstellung der Traumszenen in den Dionysiaka auch den Traum des Dionysos im 10. Buch anführt (Nonn. D.10,264-266). Gianfranco Agosti wiederum ordnet die Dionysos-Ampelos- sowie die Kalamos-Karpos-Episode in den größeren Rahmen der Liebesgeschichten in den Dionysiaka ein, Ronald Newbold behandelt die Trauer- und Trostszenen, darunter auch die Szene um Ampelos’ Tod, Vincent Giraudet betont außerdem die Kompositionslinien zwischen der Ampelos- und StaphylosEpisode, die durch die gemeinsamen Motive Wein und Metamorphose geschaffen werden. ${ }^{74}$

Von David Hernández de la Fuente wird die Geschichte um den Satyrn als Fusion von mehreren Elementen gesehen, die vom Hellenismus inspirierte Liebesgeschichte

68 Vgl. Chuvin (1991) 137. - Auch Francis Vian verortet die Szene am Ufer des Paktolos, die mehrmalige Nennung der Landschaft Maioniens sowie der Göttin Rheia in Kleinasien; vgl. Vian (1995a) 209- 214 (561 - 564). - Für einen Überblick über Quellen und Komposition der Ampelos-Episode siehe auch Chrétien (1985) 52-54, 67-81 und Vian (2003) 3-29, 51-91.

69 Keydell (1932) 182 (494); vgl. Koehler (1853) 26.

70 Vgl. Vian (1994).

71 Vgl. Vian (1995a) $201-205$ (553-556).

72 Vgl.Vian (1995a) 206 - 209 (558 - 560); Vian konstatiert hier außerdem motivische Parallelen zum fragmentarisch erhaltenen Satyrspiel Dionysiskos des Sophokles. - Zur Debatte um Nonnos' Verhältnis zu Claudian vgl. Braune (1948); Haidacher (1949) 81-87; Duc (1994) 34-43; Boscarino (1997); Newbold (2001). Zu Nonnos und Ovid siehe Braune (1935); Haidacher (1949) 55 - 63; Herter (1981); Schulze (1985); Paschalis (2014). Zu Nonnos und Nemesian siehe Magaña Orúe (1997); Salanitro (1997). Auch Hollis (2006) 141f. Anm. 3 zweifelt am direkten Einfluss der Lateiner auf Nonnos, der Grund liege in einem „common use of Hellenistic models“; vgl. außerdem Knox (1988); Simon (1999) 29 - 40; Cameron Alan (2011) 641f. - Zum Einfluss des Lateinischen im spätantiken östlichen Mittelmeerraum allgemein siehe Cameron Averil (2009); Evans T. V. (2012).

73 Vgl. Gigli Piccardi (1985) 40f., 46, 53-55, 61, 65, 68-72.

74 Vgl. Auger (2003) 416; Agosti (2004) 464; Newbold (2003-2005) 149f.; Giraudet (2005) $91 \mathrm{f}$. 
zwischen einem Gott und einem Sterblichen wird mit Wettkämpfen nach homerischem Vorbild verschränkt, wobei Motivwiederholungen ebenso kompositionsbildend seien wie Prophezeiungen und Vorverweise. ${ }^{75}$ Strukturbildende Funktion haben auch die Kalamos-Karpos-Geschichte und die kosmologische Ebene um die Horen und Helios. Auf die Spiegelung des Schicksals des Ampelos in der Kalamos-Karpos-Episode geht auch Robert Shorrock in seiner jüngsten Monographie ein: „This consolatory story is clearly designed to mirror and anticipate the story of Ampelus. [...] the ultimate fate of Calamus and Carpus clearly anticipates that of Ampelus: all three characters are transformed into eponymous substances (reeds, fruit and vines respectively) and so manage to escape the finality of death and live on in a new form. " ${ }^{\text {"76 }}$ Shorrock sieht in der Ampelos-Episode eine Synthese aller Genera, ${ }^{77}$ durch den geänderten Kontext, den Nonnos im Gegensatz zum homerischen Epos biete, weise der Dichter die epische zugunsten der erotischen Dichtung zurück und verwende literarische Traditionen zur Kreation eines eigenen dionysischen Epos. Auch die nonnianische Dichtungstheorie will Shorrock an den Büchern 10 - 12 ablesen, wenn er die Genese des Weinstocks und den alle Getränke vereinenden Wein als Bekenntnis zur dionysischen Dichtung liest, die ihrerseits den Anspruch erhebt, sämtliche Genera in sich zu vereinen. ${ }^{78}$ Der Genusmix äußert sich auch in der Anwendung unterschiedlicher rhetorischer Verfahren, die Laura Miguélez Cavero in der bevorzugten Darstellung von Farben, Geräuschen und Gerüchen sowie in der bildhaften und plastischen Ausgestaltung der Erzählung begründet sieht, durch die beim Leser möglichst alle Sinne angesprochen werden sollen. ${ }^{79}$

\section{Religiöse Aspekte}

Neben der Problematik der Komposition stellten die Fragen nach der Konfessionszugehörigkeit des Autors und der religiösen Ausrichtung seines Werkes bis in jüngste Zeit viel diskutierte Inhalte der Nonnos-Forschung dar, die auch in der AmpelosEpisode greifbar werden: Für Glen Bowersock ist Dionysos zweifelsfrei im Sinne eines christlichen Erlösers zu verstehen und die soteriologischen Elemente sind im Rahmen spätantiker synkretistischer Tendenzen erklärlich; die Vereinigung heidnischer und christlicher Züge im Protagonisten Dionysos diene der Konzeption eines heidnischen Epos, das in den Kernbereichen einige wesentliche Aspekte der neuen Religion ab-

75 Vgl. Hernández de la Fuente (2008) 57 - 62, 80-86.

76 Shorrock (2011) 99; vgl. auch Cribiore (2001) 209; Miguélez Cavero (2008) 143.

77 Vgl. Shorrock (2001) 132-137.

78 Shorrock (2001) 137: ,Just as Ampelus dies and is brought back to life through wine, so Nonnus' erotic narrative (which dies with Ampelus) is not extinguished without trace, but is brought back to life as an essential component of the new Dionysiac poetic.“

79 Vgl. Miguélez Cavero (2008) 126, 130-135. - Zur Forschungsgeschichte der Rhetorik und der hellenistischen Dichtung siehe S. 120 f. sowie Kap. 6.2. 
sorbiert habe. ${ }^{80}$ Relativiert wird Bowersocks These von Wolfgang Liebeschuetz, der das Weinen des Dionysos in Nonn. D. 12,171 nicht notwendigerweise christlich-soteriologisch verstanden wissen will ${ }^{81}$ und trotz der christlichen Position des Autors keineswegs eine Verchristlichung heidnischer Traditionen sieht. In diesem Zusammenhang verwirft Liebeschuetz auch die in der Nonnos-Forschung lange vertretene Vorstellung der Konversion des Autors zum Christentum zugunsten einer Areligiosität, die das heidnische Epos in einem weitgehend christianisierten Umfeld einzubetten vermag. ${ }^{82}$

Aktuelle Arbeiten sehen Nonnos' Epen als Ergebnis der Symbiose von Heiden- und Christentum im spätantiken Ägypten. Die Ampelos-Episode biete, so Robert Shorrock, Parallelen einerseits zu Tod und Auferweckung des Lazarus, andererseits zu Tod und Auferstehung Christi in der Paraphrase ${ }^{83}$ vor allem in der Metamorphose des Ampelos und im Vergleich Jesu mit dem Weinstock sei eine Entsprechung zu finden, ${ }^{84}$ ebenso in den Tränen, die Jesus und Dionysos vergießen (Nonn. P.11,123f.; Nonn. D.12,171f.). Zu beobachten seien außerdem für die spätantike Gesellschaft insgesamt charakteristische eschatologisch-soteriologische Grundtendenzen, ${ }^{85}$ die besonders christliches Gedankengut transportieren und Ampelos' physische Transformation in den Weinstock im Rahmen der spätantiken theologischen Diskussion über die Eucharistie verständlich machen würden. Im Zusammenhang mit Nonn. D. 11,316 und Nonn. P. 15,1-19 arbeitet Daria Gigli Piccardi als weiteren religiösen Aspekt die Einflüsse der dionysischen Mysterien auf die Geschichte um Dionysos und den jungen Satyrn heraus, ${ }^{86}$ die sich ähnlich wie das Christentum mit Fragen des Lebens und der Auferstehung nach dem Tod auseinandersetzen und das zeitgenössische Publikum in seinem Interesse an mythischer Erlösungserwartung bedienen.

\subsection{Aufgaben und Zielsetzungen}

Trotz der Einbeziehung der Ampelos-Episode in die Forschungen zur Gesamtkomposition der Dionysiaka liegt bislang keine eingehende Untersuchung dieser Episode vor. Die Bemerkungen zur Episode betreffen oftmals nur einzelne ausgewählte Text-

80 Vgl. Bowersock (1990) $44 \mathrm{f}$.

81 Vgl. Liebeschuetz (1996) 82.

82 Vgl. Liebeschuetz (1996) 82 f.; vgl. Marcellus (1856) III: „[...] ce païen, esprit fort, qui cède, dans le sein de la Thébaïde, à l'influence naissante du christianisme, et fait résonner sur sa lyre toute vibrante encore des origines bachiques les récits du chaste disciple.“

83 Vgl. Shorrock (2011) 100.

84 Shorrock (2011) 100: „It seems hard to deny that there is a prominent overlap - and suggestive interplay - between the two texts: where Christ uses metaphor to describe his own similarity to a vine, in the Dionysiaca, the young satyr called Ampelus is actually transformed into the plant that bears his name; Christ the metaphorical vine will suffer death, but will be bodily resurrected and will then return to heaven; Ampelus, the satyr, will die, but will enjoy resurrection and new life as the vine.“

85 Vgl. Shorrock (2011) 100-108.

86 Vgl. Gigli Piccardi (2003) 732 - 736, 783 f.; Caprara (2008). 
stellen oder bieten lediglich generalisierende Aussagen, ohne auf konkrete textliche Details einzugehen. Zahlreiche Fragen sind bislang offen geblieben: Hat Nonnos Vorbilder für die Gestaltung der sonst so unbekannten Ampelos-Figur, und wenn ja, welche? Warum setzt er die Liebesgeschichte um Dionysos und den Satyrn gerade an diese Stelle im Epos? Welche Rückschlüsse auf die Poetik des gesamten Epos lässt die Detailinterpretation einer ausgewählten Episode zu? Auf der Grundlage der bisherigen Forschungsansätze will die vorliegende Arbeit einen umfassenden Einblick in die Figurengestaltung, die Verarbeitung literarischer Traditionen und die Komposition der Ampelos-Episode geben sowie ihre Relation zu anderen Passagen der Dionysiaka aufzeigen, wobei der Fokus auf die folgenden vier Bereichen gerichtet wird: (1.) den Ampelos-Mythos, (2.) den Umgang mit den literarischen Traditionen, (3.) den inneren Aufbau der Ampelos-Episode und deren Einbettung ins Epos-Ganze sowie (4.) die besonderen Merkmale nonnianischer und spätantiker Poetik. Der Ausschnitt aus Nonnos' epischem Werk wird vor dem Hintergrund des spätantiken Literatur- und Kunstschaffens gesehen und in den sozio-kulturellen Kontext der hellenischen Bildungselite Ägyptens eingeordnet. Mit der Gegenüberstellung von Ausschnitten aus der Ampelos-Episode, von Szenen der Ampelos-Episode mit Auszügen aus anderen Büchern der Dionysiaka wie auch mit Texten anderer griechischer oder römischer Autoren werden, diachron von Homer bis in die Spätantike, intra- und intertextuelle Bezüge herausgearbeitet.

Kapitel 2 illustriert anhand der Eingangsszene der Ampelos-Episode, mit welchen sprachlichen, literarischen und motivischen Mitteln Nonnos auf sein narratives Ziel, die Genese des Weinstocks und die Initialisierung des Weinkultes, hinarbeitet. Kapitel 3 und 4 behandeln den Ampelos-Mythos und zeigen den jungen Satyrn im Gefolge des Dionysos, der zum Namensgeber für die neue Pflanze und neben dem Weingott selbst zum Hauptakteur in den Büchern 10 - 12 wird: Kapitel 3 verfolgt die literarischen Spuren zu Ampelos außerhalb der Dionysiaka sowie in der bildenden Kunst und zeichnet das nonnianische Ampelos-Porträt nach, Kapitel 4 arbeitet die einzelnen mythischen Versatzstücke und literarischen Topoi für den sonst unbekannten Ampelos heraus.

In Kapitel 5 wird dargelegt, wie neben Homer auch die hellenistischen Dichter und verschiedene weitere Genera Eingang in die Dionysiaka fanden. Gefragt wird insbesondere, inwiefern Nonnos die Eigentümlichkeiten der unterschiedlichen Genera adaptiert und sich diese für seine Poetik kreativ zunutze macht. Während die Einflüsse der antiken Rhetorik, namentlich der Synkrisis, der Ekphrasis und des Enkomions, auf die Konzeption der Ampelos-Episode in Kapitel 6 thematisiert werden, bietet Kapitel 7 eine umfassende Darstellung des inneren Aufbaus und der Komposition sowie der Anbindung an die übrigen Bücher der Dionysiaka. Durch die so gewonnenen Erkenntnisse im Kleinen sollen Rückschlüsse auf die Kompositionsprinzipien der Dionysiaka in ihrer Gesamtheit gezogen werden. Kapitel 8 dient sowohl der Zusammenfassung der Ergebnisse als auch dem Ausblick auf weitere Forschungsfelder, welche die Dionysiaka im Licht ihres spätantiken sozialen und kulturellen Umfeldes sehen und die Frage nach einer möglichen christlichen Lesart aufwerfen; die dabei prä- 
sentierten Exempel nonnianisch-spätantiker Dichtkunst erheben keinerlei Anspruch auf Vollständigkeit im Sinne der Formulierung einer umfassenden Poetik der Spätantike. Der Hinweis auf sprachlich-stilistische, kompositionelle und kulturhistorische Parallelen der Ampelos-Episode zu anderen literarischen und künstlerischen Zeugnissen soll vielmehr eine universelle Perspektive auf das spätantike Literatur- und Kunstschaffen eröffnen.

Die in der Arbeit zitierten griechischen Textpassagen der Dionysiaka folgen der französischen Les-Belles-Lettres-Edition, die unter der Ägide Francis Vians in einem mehr als drei Jahrzehnte umfassenden Projekt entstand ${ }^{87}$ die deutschen Übersetzungen der im Buch ausführlich behandelten Textpassagen der Dionysiaka sowie weiterer griechischer Texte stammen, so nicht anders vermerkt, von der Verfasserin und erheben keinerlei poetischen Anspruch, sondern wollen durch die möglichst wortgetreue Prosawiedergabe das Fundament für die anschließenden sprachlichen und literarischen Analysen legen. ${ }^{88}$

87 Siehe dazu die Bibliographie.

88 Neben den beiden deutschen Übersetzungen der Dionysiaka sind insbesondere die beiden italienischen, Del Corno u. a. (1997) und Gigli Piccardi (2003) sowie die englische von Rouse und die spanische von Manterola u. Pinkler (1995) zu nennen, die jeweils auch die Ampelos-Episode enthalten. Übersetzungen von Auszügen der Episode liefern außerdem Appel (1994) (polnisch) sowie ein Anonymus in „The London Magazine“ von 1822, der unter dem Pseudonym Vida die Wettspiele zwischen Dionysos und Ampelos, das unmittelbare Geschehen vor dem Sturz des Satyrn sowie dessen Tod und die Trauer des Weingottes in englischer Übersetzung bietet, vgl. Vida (1822) und (1822a). 